\title{
FABRICATION AND CHARACTERIZATION OF THIN FILMS OF THE ORGANIC SUPERCONDUCTOR $\alpha_{\mathrm{t}-}(\mathrm{BEDT}-\mathrm{TTF})_{2} \mathrm{I}_{3}$
}

J. Moldenhauer, H. Wachtel, D. Schweitzer

3. Physikalisches Institut, Universität Stuttgart Pfaffenwaldring 57, 70550 Stuttgart, Germany

B. Gompf, W. Eisenmenger

1. Physikalisches Institut, Universität Stuttgart Pfaffenwaldring 57, 70550 Stuttgart, Germany

P. BELE, H. BRUNNER

MPI für medizinische Forschung, AG: Molekülkristalle Jahnstr. 29, 69120 Heidelberg, Germany

AND H.J. KELLER

Anorganisch-Chemisches Institut, Universität Heidelberg Im Neuenheimer Feld 269, 69120 Heidelberg, Germany

$\alpha$-(BEDT-TTF $)_{2} \mathrm{I}_{3}$ is a quasi-two-dimensional organic metal with a metal-insulator phase transition at $135 \mathrm{~K}$. Thermal treatment at about $80^{\circ} \mathrm{C}$ leads to the metallic system $\alpha_{\mathrm{t}}$-(BEDT-TTF) ${ }_{2} \mathrm{I}_{3}$, which becomes superconducting below $8 \mathrm{~K}$. Thin films of the $\alpha$-phase (thickness between 500 and $3000 \AA$ ) have been evaporated in high vacuum onto several substrates and characterized by means of X-ray diffraction, scanning electron microscopy, atomic force microscopy and low field microwave absorption. Depending on the temperature of the substrate and the evaporation rate, the films exhibit different degrees of microcrystallinity, which under certain conditions can be strongly reduced and a completely covering film can be obtained. X-ray diffraction spectra reveal a high orientation with the $c$-axis perpendicular to the substrate and as well the successful conversion into the $\alpha_{t}$-phase by tempering. Scanning electron microscopy and atomic force microscopy investigations prove that the conversion takes place without reducing the mechanical quality of the films. Low-field microwave-absorption experiments show that the $\alpha_{\mathrm{t}}$-films become superconducting with an onset at $9 \mathrm{~K}$.

PACS numbers: 74.70.Kn, 73.50.-h, 81.15.Gh 


\section{Introduction}

During the last decade organic conductors and superconductors have attracted considerable interest. Aside from the interesting physics of low-dimensional systems, in the area of so-called molecular electronics some effort is made to scale down electronic circuits and devices to dimensions of a few unit cells or even molecules. Radical cation salts of the organic donor BEDT-TTF (bis(ethylene-dithiolo)tetrathiafulvalene) exhibit a great variety in their transport properties from semiconducting to metallic and even superconducting behaviour with $T_{\mathrm{c}}$ up to $12.4 \mathrm{~K}$.

A most interesting and well characterized system is the $\alpha$-phase of (BEDT-TTF $)_{2} \mathrm{I}_{3}$. At room temperature it is a metal with a conductivity of 60-200 S/cm and shows a sharp metal-insulator transition at $135 \mathrm{~K}[1,2]$. This phase can be transformed (at $75^{\circ} \mathrm{C}$ for $2-3$ days) into the metallic $\alpha_{\mathrm{t}}$-phase, which becomes superconducting below $8 \mathrm{~K}[3,4]$.

\section{Experimental}

The radical cation salts were evaporated in a vacuum of $10^{-8} \mathrm{mbar}$ base pressure at temperatures between $150^{\circ} \mathrm{C}$ and $220^{\circ} \mathrm{C}$. The source was between 30 and $5 \mathrm{~cm}$ away from substrates kept at temperatures between $77 \mathrm{~K}$ and $460 \mathrm{~K}$. Rates of deposition were varied between 0.2 and $3 \mathrm{~A} / \mathrm{s}$. Substrates were silicon, glass, mica and HOPG. When the temperature of the substrate was below $300 \mathrm{~K}$, they were heated after the evaporation process at a rate of $5-15 \mathrm{~K} / \mathrm{h}$.

\section{Results \\ 3.1. Films of $\alpha-(B E D T-T T F)_{2} I_{3}$}

First attempts in evaporating thin films onto silicon and glass substrates were performed at substrate temperatures of room temperature (RT) and above. At these temperatures the sticking coefficient on the substrate of the incident molecules is small, the evaporation rate has to be high and the source-target distance should be only a few $\mathrm{cm}$. This method of evaporating (BEDT-TTF) ${ }_{2} \mathrm{I}_{3}$ onto substrates, which has already been applied [5,6], yields results as shown in Fig. 1 (left picture), i.e. a film consisting of many crystals with $1-3 \mu \mathrm{m}$ size and a not completely covered substrate surface.

An extensive investigation of another route - which mainly consists of a lower substrate temperature - with varying source-target distances and evaporation rates led to much better results, as shown in Fig. 1 (right picture), where the surface is relatively smooth and completely covered.

For the determination of the crystal phase and the orientation of the unit cell with respect to the substrate plane, X-ray diffraction spectra of a film as shown in Fig. 1 (right) were recorded. The spectrum shown in Fig. 2 (upper part) reveals $(00 n)$ reflections known from the crystallographic data of the $\alpha$-phase, which means that the $c$-axis of the unit cell is perpendicular to the substrate plane.

For a determination of the homogeneity of the orientation rocking curves were recorded, one of which is shown in Fig. 3. The small FWHM of $1.1^{\circ}$ indicates a very good orientation of the crystalline regions of the film. 

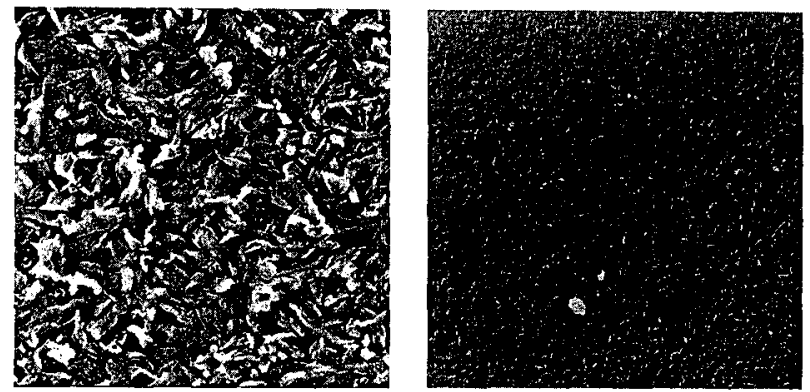

Fig. 1. SEM pictures of films of $\alpha$-(BEDT-TTF $)_{2} \mathrm{I}_{3}$ evaporated on substrates at $300 \mathrm{~K}$ (left) and $170 \mathrm{~K}$ (right). Total area of each picture is $16 \mu \mathrm{m} \times 16 \mu \mathrm{m}$ each.
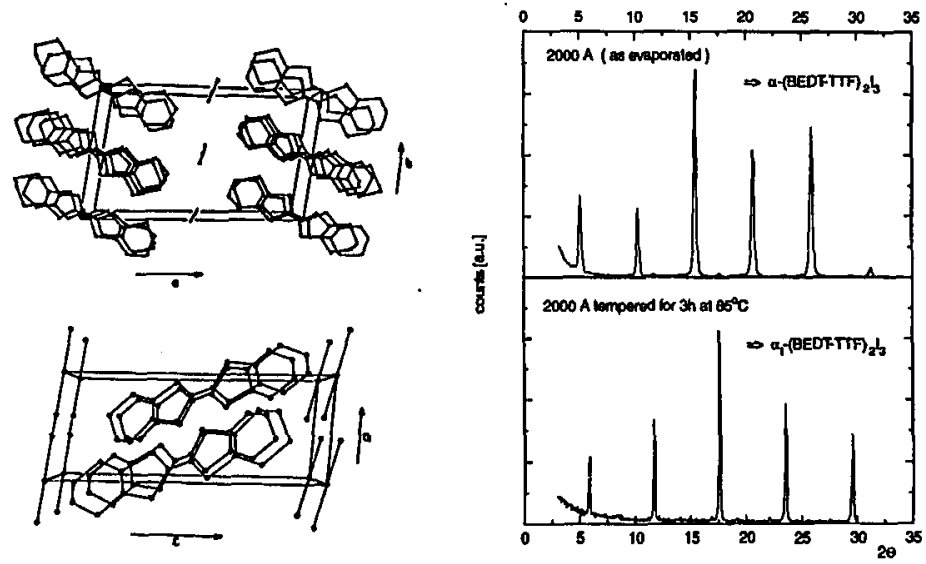

Fig. 2. Unit cells and X-ray diffraction spectra of an evaporated (above) and an afterwards tempered film (below).

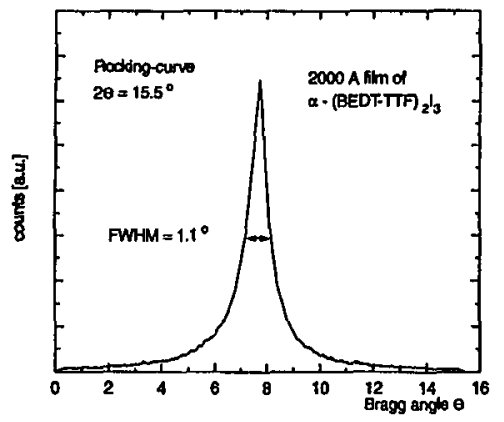

Fig. 3. Rocking-curve of an evaporated film of $\alpha$-(BEDT-TTF $)_{2} \mathrm{I}_{3}$. 


\subsection{Films of $\alpha_{\mathrm{t}}-(B E D T-T T F)_{2} I_{3}$}

The transformation of crystals of $\alpha$-(BEDT-TTF $)_{2} \mathrm{I}_{3}$ into $\alpha_{\mathrm{t}}$-(BEDT-TTF $)_{2} \mathrm{I}_{3}$ usually is performed at $75^{\circ} \mathrm{C}$ for $2-3$ days, after which the surface of the crystals is corrugated through a loss of iodine. For films of about $2000 \AA$ thickness, best results were obtained at higher temperatures, i.e. $85-90^{\circ} \mathrm{C}$ for 1.5 to 3 hours. Figure 2 (lower part) shows the X-ray diffraction spectrum of such a converted film and - as expected - $(00 n)$ reflections of the $\beta_{\mathrm{H}}$-phase (which is assumed to be equal to the $\alpha_{\mathrm{t}}$-phase) are observed. The investigations of the influence of this thermal treatment on the quality of the films with atomic force microscopy (see Fig. 4) show minor changes during the first 2 hours of exposure to elevated temperatures (Fig. 4, upper right). Longer exposure leads to the formation of microcrystals and to the evolution of uncovered areas on the substrate (Fig. 4, lower part).
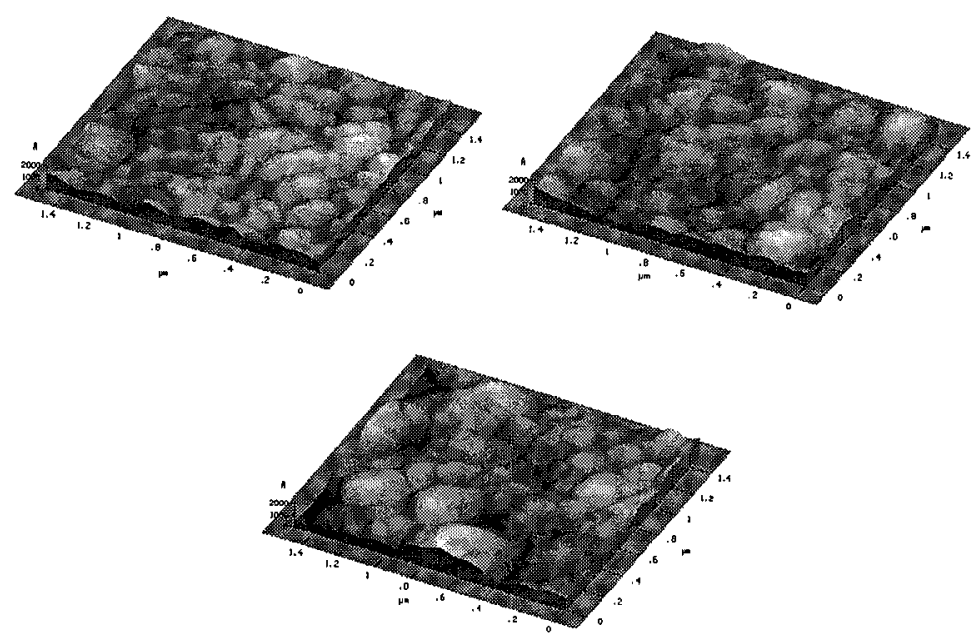

Fig. 4. AFM pictures of films: as evaporated (upper left), 1.5 hours (upper right) and 3.5 hours (below) tempered (total area of each picture is $1.5 \mu \mathrm{m} \times 1.5 \mu \mathrm{m}$ ).

\subsection{Conductivity and superconductivity}

Measurements of the dc conductivity with the standard 4-point method revealed thermally activated behaviour of the films. This might be due to the fact that the conductivity is governed by the presence of micro-crystals with grain boundaries and imperfections. This assumption is supported by the results of the low-field microwave absorption measurements, a method that has been applied to granular samples of $\mathrm{YBa}_{2} \mathrm{Cu}_{3} \mathrm{O}_{7}[7,8]$. For a proof of superconductivity in the films, we measured the microwave absorption of a film in the resonator of an $\mathrm{X}$-band microwave bridge with a field of $20 \mathrm{G}$ perpendicular to the substrate plane. On lowering the temperature, one observes the onset of the absorption signal at $9 \mathrm{~K}$ (Fig. 5, lower left), which is ascribed to a microwave loss mechanism in intergranular Josephson junctions $[7,8]$. In addition the typical microwave absorption 

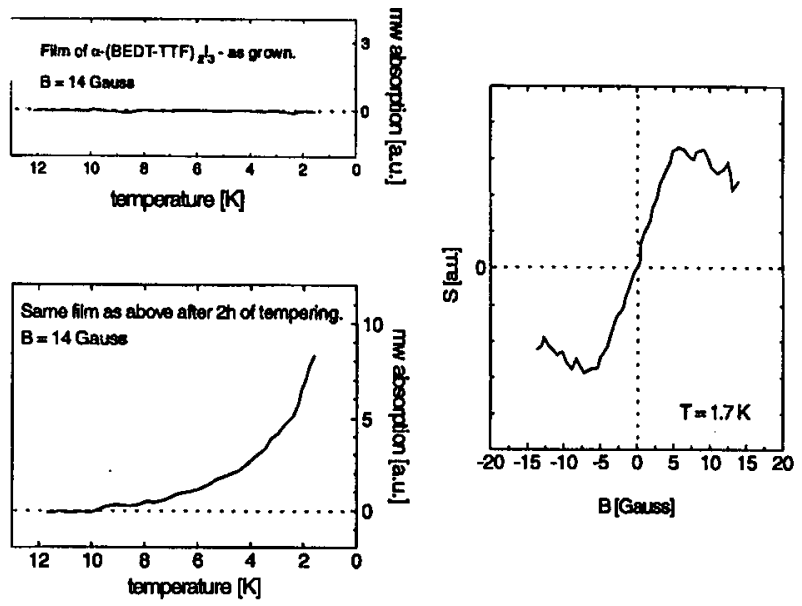

Fig. 5. Microwave absorption as a function of temperature of an $\alpha$-(BEDT-TTF $)_{2} \mathrm{I}_{3}$ film (upper left) and an $\alpha_{\mathrm{t}}$-(BEDT-TTF) ${ }_{2} \mathrm{I}_{3}$ film (lower left) and as a function of applied field (right) for an $\alpha_{t}-(B E D T-T T F)_{2} I_{3}$ film.

signal as a function of the applied field at $1.7 \mathrm{~K}$ (i.e. in the superconducting) state is shown (right part of Fig. 5).

\section{Conclusions}

Completely covering films of $\alpha_{\mathrm{t}}$ (BEDT-TTF $)_{2} \mathrm{I}_{3}$ on substrates as silicon, quartz glass, mica and graphite can be obtained by evaporation with an onset for superconductivity at $9 \mathrm{~K}$.

\section{References}

[1] K. Bender, K. Dietz, H. Endres, H.W. Helberg, I. Hennig, H.J. Keller, W. Schäfer, D. Schweitzer, Mol. Cryst. Liq. Cryst. 107, 45 (1984).

[2] K. Bender, I. Hennig, D. Schweitzer, K. Dietz, H. Endres, H.J. Keller, Mol. Cryst. Liq. Cryst. 108, 359 (1984).

[3] G.O. Baram, L.I. Buravov, L.C. Degtariev, M.E. Kozlov, V.N. Laukhin, E.E. Laukhina, V.G. Orischenko, K.I. Pokhodnia, M.K. Scheinkman, R.P. Shibaeva, E.B. Yagubskii, JETP Lett. 44, 293 (1986).

[4] D. Schweitzer, P. Bele, H. Brunner, E. Gogu, U. Haeberlen, I. Hennig, T. Klutz, R. Swietlik, H.J. Keller, Z. Phys. B, Condens. Matter 67, 489 (1987).

[5] K. Kawabata, K. Tanaka, M. Mizutani, Solid State Commun. 74, 83 (1990).

[6] K. Kawabata, K. Tanaka, M. Mizutani, Adv. Mater. 3, 157 (1991).

[7] A. Dulcic, B. Ravkin, M. Pozek, Europhys. Lett. 10, 593 (1989).

[8] M. Pozek, A. Dulcic, B. Ravkin, Physica C 169, 95 (1990). 